\title{
Aproximaciones al estado de situación geopolítica actual. Nuevos actores, conflictos y escenarios para la construcción de un orden mundial multipolar
}

\section{Approaches to the current state of the geopolitical situation. New social actors, conflicts and scenarios for a multipolar world order construction}

\author{
Juan Sebastián Schulz \\ Instituto de Investigaciones en Humanidades y Ciencias Sociales / \\ Universidad Nacional de La Plata-CONICET, Argentina \\ jsschulz@gmail.com
}

\section{Resumen:}

A partir de la Segunda Guerra Mundial, las potencias vencedoras comenzaron un proceso de construcción de un nuevo orden internacional, que posteriormente fue configurando la unipolaridad estadounidense como centro de gravedad global. Este nuevo orden se tradujo en el peso que obtuvieron los países centrales en los organismos internacionales de carácter político y económico. La crisis financiera global, desatada en 2008, significó un quiebre en las relaciones de fuerza internacionales, generó una parálisis en las economías centrales y, paralelamente, un proceso de crecimiento económico y articulación política de las economías emergentes agrupadas en los "BRICS". Este trabajo pretende ser un aporte a los estudios sobre el estado del mundo en el Siglo XXI, a partir de la recopilación y análisis de los estudios teóricos precedentes y, a su vez, plantear algunas consideraciones propias organizadas en dos ejes fundamentales: por un lado, el proceso de transnacionalización de la economía global y de consolidación de la unipolaridad estadounidense y, por el otro, la crisis financiera de 2008 y el análisis de las condiciones particulares de los países emergentes desde la perspectiva multipolar. Los resultados pretenden convertirse en objeto de debate a efectos de realizar nuevas miradas de las perspectivas sociológicas sobre los aspectos socio territoriales globales.

Palabras Clave: Geopolítica, Nuevo Orden Mundial, Multipolarismo, Emergentes, BRICS.

\section{Abstract:}

After the Second World War, the victorious powers began a process of building a new international order, which later became the American unipolarity as a center of global gravity. This new order tipped the balance in favour of the central countries, in the new international organizations of political and economic nature. The global financial crisis, unleashed in 2008, meant a break in international power relations, generated a paralysis in the central economies and, at the same time, a process of economic growth and political articulation of the emerging economies grouped as the 'BRICS'. This work aims to be a contribution to studies on the state of the world in the XXI Century, from the collection and analysis of previous theoretical studies and, in turn, raise my own considerations organized in two fundamental axis: on one hand the process of transnationalization of the global economy and consolidation of the American unipolarity and, on the other, the financial crisis of 2008 and the analysis of the particular conditions of the emerging countries from a multipolar perspective. The results are intended to become the subject of debate in order to make new views of sociological perspectives on the global socioterritorial aspects

KEYWORDS: Geopolitics, New World Orde, Multipolarism, Emerging, BRICS.

\section{INTRODUCCIÓN}

La crisis financiera global desatada en 2008 significó un quiebre de las relaciones de fuerza internacionales, al punto que resquebrajó y puso en cuestión el orden mundial que emergió como consecuencia de la Segunda Guerra Mundial, donde Estados Unidos, Gran Bretaña y Francia aparecieron como los grandes ganadores y ordenadores mundiales y de los sucesos posteriores que fueron configurando la unipolaridad estadounidense como centro de gravedad global. Este nuevo orden mundial establecido entre 1944 y 1973 se tradujo en el peso que obtuvieron las llamadas potencias centrales en los organismos internacionales de carácter político, 
como el Consejo de Seguridad de las Naciones Unidas, de carácter económico, como el Fondo Monetario Internacional, el Banco Mundial y la Organización Mundial del Comercio, además del establecimiento del G7 como espacio informal de coordinación de los asuntos mundiales. ${ }^{1}$

La crisis global de 2008 produjo una parálisis en las economías centrales, que vieron estancarse sus PBI mientras que, paralelamente, fueron ganando peso las economías emergentes, que a partir de su crecimiento económico primero, y su capacidad de articulación política después, comenzaron a plantear nuevos desafíos en el orden mundial, mostrándose críticos del ordenamiento unipolar global y esbozando lineamientos para superarlo.

El artículo se organiza en base a dos ejes fundamentales; por un lado, una caracterización del proceso de transnacionalización de la economía global, que configuró la unipolaridad anglosajona luego de la Segunda Guerra Mundial y, por otro, de los sucesos producidos a partir de la crisis financiera de 2008, centrando el análisis en las condiciones particulares de los cinco países emergentes agrupados los BRICS, ${ }^{2}$ su heterogeneidad, su arquitectura financiera y productiva y los planteos escépticos sobre su situación actual. Para ello se realiza una pormenorizada revisión de la bibliografía disponible y se plantean consideraciones propias elaboradas sobre la base de los datos recopilados. Los resultados pretenden convertirse en objeto de debate a efectos de realizar nuevas miradas de las perspectivas sociológicas.

\section{LA CONSTRUCCIÓN DE LA UNIPOLARIDAD ANGLOSAJONA Y EL PROCESO DE TRANSNACIONALIZACIÓN DE LA ECONOMÍA GLOBAL}

A partir de los años 90, con la "caída" de la URSS, se observa un proceso de profundización del proyecto de globalización financiera neoliberal, encabezado por la administración del demócrata Bill Clinton en Estados Unidos (1993-2001) y del laborista Tony Blair (1997-2007) en el Reino Unido. Este proceso, al traer aparejado una transnacionalización a escala global del capital, implicó la conformación de una nueva territorialidad que comenzó a poner en crisis al Estado-Nación como forma de organización política, económica, social y cultural. ${ }^{3}$

En 1999, la gestión demócrata de Clinton aprobó la "Ley de Modernización de los Servicios Financieros", con la cual los bancos de inversión, los fondos financieros de inversión global, las aseguradoras de riesgo y los brokers financieros quedaban libres de los controles que les había impuesto F. D. Roosevelt y la Ley Glass-Steagall de 1933, controles que habían sido elaborados para prevenir las acciones que terminaron produciendo la crisis de 1929 y que causarán, posteriormente, la crisis del 2008.

Al eliminar las restricciones a la regulación de la banca de inversión y de la especulación, se creó el terreno fértil para la proliferación de burbujas financieras, donde las grandes redes transnacionales, con mayor capacidad de apropiación de la riqueza ficticia, terminaron por apropiarse de los capitales de menor escala. Este proceso significó la multiplicación de activos ficticios, a partir de la valorización del interés emergido del proceso de especulación, sin asiento en la economía real. Las grandes bancas de inversión transnacionales dejaron cada vez más de financiar la inversión real o el comercio internacional de bienes, generando un corrimiento cada vez más marcado hacia los activos financieros, mucho más lucrativos. El avance del proyecto estratégico neoliberal global provocó la profundización no solo de la desigualdad y de la marginación de los sectores populares, sino de otras formas de capital más débiles que vieron peligrar sus intereses de acumulación de ganancias (Formento, 2015).

\section{LA CRISIS FINANCIERA GLOBAL}

La crisis de 2008 no fue una crisis más, sino que marcó un quiebre en el sistema de relaciones de poder que había ordenado el mundo luego de la Segunda Guerra Mundial y la caída de la Unión Soviética, y brindó las condiciones de posibilidad para que nuevos actores, que emergían subordinados, se fortalecieran 
y reclamaran por la configuración de un nuevo ordenamiento global.

La crisis financiera global apareció en principio como resultado del estallido de la burbuja inmobiliaria o de las hipotecas subprime. La acción que definió, profundizó y generalizó lacrisis fue la decisión del Secretario del Tesoro Henry Paulson de dejar caer a una de las mayores bancas financieras de inversión global, la Lehmann Brothers, el 15 de septiembre de 2008. Este hecho paralizó el sistema financiero global y puso en marcha la lucha-transición hacia la configuración del nuevo orden global (Formento y Merino, 2011). Esta paralización afectó, principalmente, a las economías centrales, pero permitió crecer considerablemente a las nuevas economías emergentes.En el Cuadro N. ${ }^{\circ} 1$ se observa que entre 2001 y 2009 el PBI global creció en todos los años por encima de las economías centrales (a excepción de Francia en 2001 y Reino Unido entre 2001 y 2003).

CUADRO N..$^{\circ}$.

Evolución del PBI entre 2001 y 2009 en el mundo y en las principales potencias occidentales

\begin{tabular}{|l|c|c|c|c|c|c|c|c|c|c|}
\hline & $\mathbf{2 0 0 1}$ & $\mathbf{2 0 0 2}$ & $\mathbf{2 0 0 3}$ & $\mathbf{2 0 0 4}$ & $\mathbf{2 0 0 5}$ & $\mathbf{2 0 0 6}$ & $\mathbf{2 0 0 7}$ & $\mathbf{2 0 0 8}$ & $\mathbf{2 0 0 9}$ & $\begin{array}{c}\mathbf{\%} \\
\text { Evolución } \\
\mathbf{0 1}-\mathbf{0 9}\end{array}$ \\
\hline Estados Unidos & $1 \%$ & $1,8 \%$ & $2,8 \%$ & $3,8 \%$ & $3,3 \%$ & $2,7 \%$ & $1,8 \%$ & $-0,3 \%$ & $-2,8 \%$ & $1,57 \%$ \\
\hline Alemania & $1,7 \%$ & $0 \%$ & $-0,7 \%$ & $1,2 \%$ & $0,7 \%$ & $3,7 \%$ & $3,3 \%$ & $1,1 \%$ & $-5,6 \%$ & $0,6 \%$ \\
\hline Francia & $2 \%$ & $1,1 \%$ & $0,8 \%$ & $2,8 \%$ & $1,6 \%$ & $2,4 \%$ & $2,4 \%$ & $0,2 \%$ & $-2,9 \%$ & $1,16 \%$ \\
\hline Japón & $0,4 \%$ & $0,3 \%$ & $1,7 \%$ & $2,4 \%$ & $1,3 \%$ & $1,7 \%$ & $2,2 \%$ & $-1 \%$ & $-5,5 \%$ & $0,39 \%$ \\
\hline Reino Unido & $2,7 \%$ & $2,5 \%$ & $4,3 \%$ & $2,5 \%$ & $2,8 \%$ & $3 \%$ & $2,6 \%$ & $-0,3 \%$ & $-4,3 \%$ & $1,76 \%$ \\
\hline Mundo & $\mathbf{1 , 8} \%$ & $\mathbf{2 , 1} \%$ & $\mathbf{2 , 8} \%$ & $\mathbf{4 , 1} \%$ & $\mathbf{3 , 6} \%$ & $\mathbf{4 , 1} \%$ & $\mathbf{3 , 9} \%$ & $\mathbf{1 , 5} \%$ & $-\mathbf{2 , 1} \%$ & $\mathbf{2 , 4 3} \%$ \\
\hline
\end{tabular}

Fuente: Elaboración propia con datos del Banco Mundial (http://datos.bancomundial.org/)

El crecimiento sostenido de las nuevas economías emergentes contrapesó el estancamiento de las potencias centrales y evitó la caída del PBI global. Países como Angola (su PBI creció en promedio un 12,4\% anual entre 2001 y 2009), China (crecimiento del 10,5\% en el mismo período), Nigeria (9,3\%), India (7,2\%), Cuba (5,5\%), Arabia Saudita (5,1\%), Rusia (4,9\%), Irán (4,8\%), formaron parte de este conjunto de países. Como señalan Gobbi y Grande (2013, p. 5),

la tendencia que se visualizaba hasta el año 2008 de que los países emergentes y en desarrollo se estaban transformado en el motor del crecimiento global, se consolidó a partir de la crisis dando un gran salto cuantitativo que las posicionó como generadoras de más del $80 \%$ del crecimiento de la economía mundial.

La crisis financiera permitió a nuevos (o no tan nuevos) actores aparecer en el escenario mundial planteando que la arquitectura global, como estaba ordenada hasta el momento, había quedado obsoleta, y que era tiempo de un nuevo orden mundial diferente del actual. Esta crisis, como en otros momentos de la historia, sirvió para poner en tela de juicio la viabilidad y legitimidad del ordenamiento global y el modelo de acumulación vigente hasta el momento, y le permitió a nuevos actores colocarse en el centro de la escena, planteándose como los nuevos motores de la recuperación (Gobbi y Grande, 2013). Como sostiene Turzi (2011), la crisis de 2008 actuó como el catalizador que le permitió a estos nuevos actores, subordinados en la vieja arquitectura financiera, pasar de un agrupamiento basado en la coincidencia de factores a un agrupamiento basado en la coordinación económica y política a partir de la convergencia de intereses.

Esto fue posible, al menos, por dos factores: en primer lugar, la crisis financiera produjo que los grandes grupos transnacionales, en la búsqueda de salvarse de la crisis, redireccionaran sus inversiones hacia los mercados emergentes. Como señala Claudio Katz (2011, p. 31), 
la reestructuración neoliberal también incluyó una significativa deslocalización de las firmas, que lucran en el exterior con menores costos salariales acentuando la pérdida de puestos de trabajo. (...) La recesión local coexiste con el aumento de las utilidades foráneas que obtienen las principales 500 firmas.

Como se observa en el Gráfico N.¹, a partir de 2009 China igualó los ingresos de Inversión Extranjera Directa (IED) de Estados Unidos, que vio emigrar casi 180 mil millones de dólares hacia otros destinos. El resto de los países del BRICS, si bien sufrieron, inevitablemente, los efectos de la crisis, vieron aumentar considerablemente el flujo de inversiones entre 2005 y 2013: en Rusia, el aumento fue del 346\%, en la India el crecimiento del flujo de inversiones fue de $287 \%$, mientras que Brasil lo hizo en un $423 \%$. Otras potencias occidentales, en tanto, como Japón y Alemania, mantuvieron índices similares de IED en 2005 y 2013. Si para el año 2000 solamente un 6\% de las inversiones de las empresas transnacionales se realizaban en los países del tercer mundo, después de la crisis financiera global esta cifra se elevó a más del 20\%.

GRÁFICO N.ำ.

Inversión extranjera directa, entrada neta de capital (balanza de pagos, en millones de US\$) 2005-2013

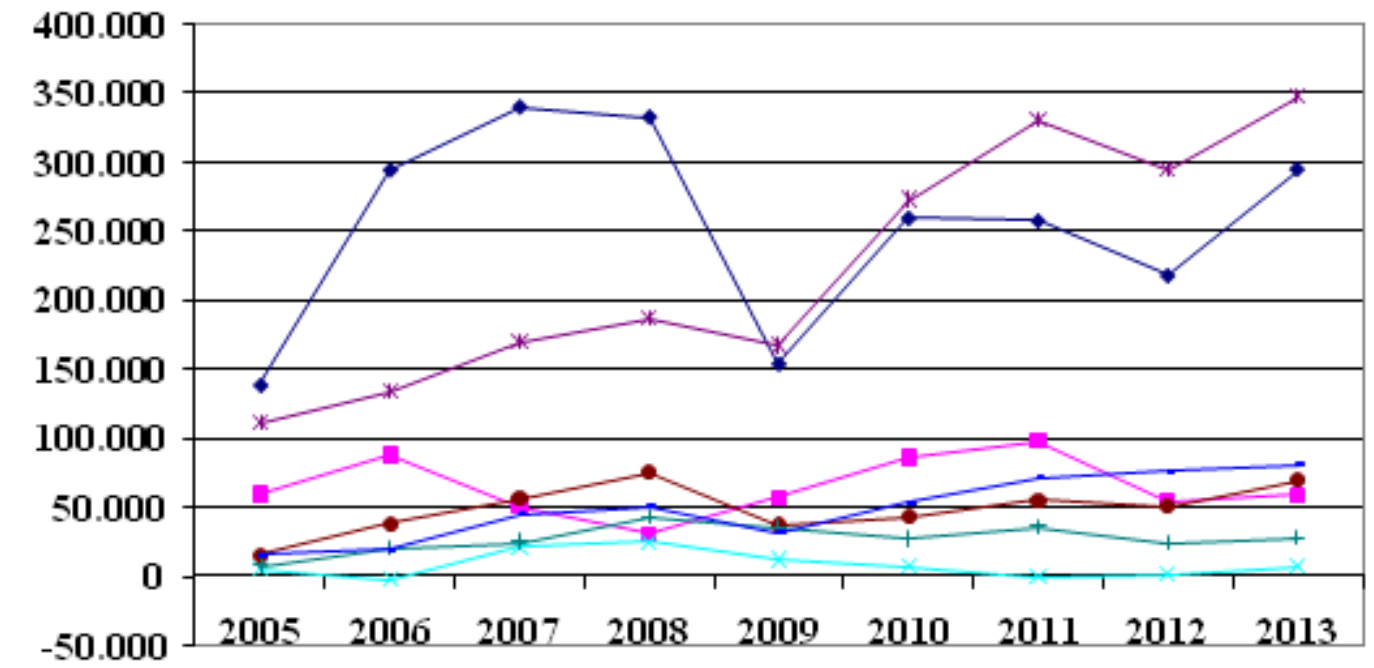

$\rightarrow-$ EstadosUnidos $\rightarrow-$ Alem ania $\longrightarrow$ Japón $\rightarrow$ China $\rightarrow$ Rusia $\multimap$ India $\longrightarrow$ Brasil

Fuente: Elaboración propia con datos del Banco Mundial

Pero esto no permitiría explicar de por sí la emergencia de nuevos actores reclamando protagonismo mundial. De hecho, entre los nuevos mercados emergentes, hubo algunos que continuaron subordinados a la lógica financiera del capital angloamericano, mientras que otros comenzaron a articular y posicionarse a favor de una nueva arquitectura financiera y de un nuevo orden global. En segundo lugar, entonces, es importante destacar que al interior de cada uno de los países emergentes se profundizó una batalla entre los proyectos estratégicos en pugna, donde lo que se puso en juego es cómo intervenir ante la crisis: mientras que los grupos financieros transnacionalizados pugnaron por subordinar a los emergentes al proyecto financiero global, los grupos económicos locales y empresas multinacionales de capital local batallaron para que la salida se dé mediante la coordinación de los países emergentes con un proyecto soberano. En este sentido, Pelfini y Fulquet (2015) identifican dos fuerzas sociales diferentes pugnando al interior de cada uno de los países de los emergentes articulados en el BRICS: por un lado, lo que los autores denominan "el clásico establishment" entendido como la clase tradicionalmente dominante y, por el otro, las "elites emergentes" o "grupos estratégicos", las cuales articulan a fracciones de clase y sectores sociales que venían estando subordinados. 
Retomando los desarrollos teóricos de Arrighi, Carlos E. Martins (2014) va a afirmar que, entre 1994 y 1998, atravesamos una fase de expansión (Fase A) del ciclo Kondratiev que se articulaba a dos momentos descendentes: el de la potencia hegemónica y del capitalismo. Esto lo observa analizando el crecimiento del PBI per cápita mundial y la tasa de ganancia, que se recupera entre 1994 y 1998, pero que luego de las crisis de 2001 y de 2008 ingresa en su última subfase de madurez, y en condiciones de agotarse. Martins afirma que a partir de allí atravesamos un ciclo descendente de la larga duración (Fase B), caracterizado por la crisis de la hegemonía estadounidense y por la crisis civilizatoria del modo de producción capitalista, a partir de la revolución científico-tecnológica. De este modo, vemos un profundo proceso de financiarización del capital, una crisis del eje atlantista de la economía mundial y el declive de las potencias marítimas. Por otro lado, el autor afirma que se produce paralelamente un desplazamiento del dinamismo hacia China y el Este Asiático, la ascensión de los regionalismos y de los hinterlands como nuevo fundamento geopolítico de la economía mundial y de la construcción de un nuevo sistema-mundo multipolar.

\section{El NUEVo PROTAGonismo DE LOS "EMERgENTES"}

Entre el conjunto de países emergentes, aquellos agrupados en el BRICS fueron los que tomaron un mayor protagonismo. Entre 2000 y 2013 su PBI pasó de representar menos del 40\% del PBI mundial a la mitad del mismo, y esta tendencia también se reflejó en el peso de sus inversiones, el consumo y las exportaciones mundiales. Los BRICS reúnen, además, $42 \%$ de la población mundial, 20,4\% de la producción y 17,6\% del comercio (Cuadro N..$^{\circ}$ ). Producto de esto, cuatro de sus miembros se encuentran ubicados entre las diez economías más grandes del planeta -China (2), India (3), Rusia (6) y Brasil (7)-.

CUADRO N. ${ }^{\circ}$.

Los BRICS en números (2014)

\begin{tabular}{|l|l|l|l|c|}
\hline & Población & Territorio & PBI (PPA) & $\begin{array}{c}\text { Crecimiento } \\
\text { PBI (\% 2010- } \\
\text { 2014) }\end{array}$ \\
\hline Brasil & 204.259 .812 hab. & $8.515 .770 \mathrm{~km}^{2}$ & uSs 3.264 .000 .000 .000 & $3,4 \%$ \\
\hline Rusia & 142.423 .773 hab. & $17.098 .242 \mathrm{~km}^{2}$ & uSs 3.745 .000 .000 .000 & $2,8 \%$ \\
\hline India & 1.251 .695 .584 hab. & $3.287 .263 \mathrm{~km}^{2}$ & uSs 7.393 .000 .000 .000 & $7,2 \%$ \\
\hline China & 1.367 .485 .388 hab. & $9.596 .960 \mathrm{~km}^{2}$ & uSs 18.031 .000 .000 .000 & $8,6 \%$ \\
\hline Sudáfrica & 53.675 .563 hab. & $1.219 .090 \mathrm{~km}^{2}$ & uSs 705.000 .000 .000 & $2,4 \%$ \\
\hline BRICS & 3.019 .540 .0120 hab. & $39.717 .325 \mathrm{~km}^{2}$ & uSs 33.138 .000 .000 .000 & - \\
\hline Estados Unidos & 321.368 .864 hab. & $9.833 .517 \mathrm{~km}^{2}$ & uSs 17.419 .000 .000 .000 & $2,2 \%$ \\
\hline Unión Europea & 513.949 .445 hab. & $4.324 .782 \mathrm{~km}^{2}$ & uSs 18.423 .000 .000 .000 & $0,6 \%$ \\
\hline Japón & 126.919 .659 hab. & $377.915 \mathrm{~km}^{2}$ & uSs 4.631 .000 .000 .000 & $1,5 \%$ \\
\hline
\end{tabular}

Fuente: Elaboración propia con datos del Banco Mundial (https://www.datos.bancomundial.org), Indexmundi (https://w ww.indexmundi.com) y The World Factbook (https://www.cia.gov/library/publications/resources/the-world-factbook/).

Distintos autores han reflexionado sobre cuál es el denominador común que permite agrupar en un mismo bloque común a este grupo de países. En este sentido, Mariano Turzi (2011) señala que, sin duda, no es la proximidad geográfica lo que explica la unión de los BRICS, ni tampoco la pertenencia a una tradición política y cultural común. Ni siquiera han compartido variables económicas similares: mientras que China y la India han experimentado tasas notables de crecimiento, las de Brasil y Rusia han permanecido más bien estancadas, incluso con tendencia a la baja. La inclusión de Sudáfrica en 2011, en tanto, puso en cuestión los postulados básicos con los que la Goldman Sachs había sintetizado en un acrónimo a este conjunto de países: no es un país con una gran extensión territorial (ocupa el lugar número 25), no ha recibido considerables cantidades de inversión extranjera directa (ocupó el lugar número 42 en 2014) y tampoco ocupa los primeros lugares respecto de su PBI (para 2013 se ubicaba en la posición número 33 a nivel global, según datos del 
FMI). No hay que buscar aquí, entonces, las condiciones de posibilidad para considerar a estos países como partes de un bloque homogéneo.

Un elemento importante a resaltar es que, para referirse a este grupo de países, la literatura académica, económica y política (así como también los propios BRICS) comienzan a utilizar el concepto de emergentes , dejando de lado las categorías tradicionales de "países subdesarrollados" o "países en vías de desarrollo", que habían caracterizado los abordajes teóricos precedentes. La categoría de "potencias emergentes" no es nueva, sino que comenzó a utilizarse en los años ochenta y noventa para analizar a los países del Este y del Sudeste de Asia, resaltando sus altas tasas de crecimiento y su gran potencial económico. De esta manera, la categoría de emergentes da cuenta de una nueva situación, al definir una dinámica de mayor incidencia en la economía global, que no se caracteriza necesariamente por un aumento en el nivel de su "desarrollo" subordinado al sistema político-financiero tradicional, sino criticando su lógica de funcionamiento y, finalmente, intentando estructurar un nuevo orden alternativo.

Sin embargo, los distintos actores le otorgan diferentes significados al concepto de emergente. El término mercado emergente fue inventado en 1981 por el economista Antoine van Agtmael, quien afirmó que estaba tratando de iniciar un fondo de inversión para el tercer mundo, para invertir en acciones de países en desarrollo, pero estaba siendo rechazado por su alto riesgo: "Trasiego mi cerebro, por fin se me ocurrió un término que sonaba más positivo y estimulante: los mercados emergentes. 'Tercer Mundo' sugería estancamiento; mientras que 'mercados emergentes' sugería progreso, levantamiento y dinamismo" ("Ins and outs", 2008). La Goldman Sachs, por su parte, hace referencia en sus informes a los BRICS (y luego también a los "Next-11" ) como "mercados emergentes" o "economías emergentes", destacando por sobre otros indicadores sus importantes tasas de crecimiento de PBI.

El acrónimo BRIC, en este sentido, lejos de representar el deseo de la Goldman Sachs por la configuración de un bloque de poder, significaba una política de aliento a sus principales accionistas, fondos de inversión y grupos transnacionales, para que redireccionen sus inversiones hacia los llamados "nuevos mercados emergentes", es decir, mercados que garantizarían una ganancia asegurada en un contexto de crisis financiera.

En los informes de la Goldman Sachs sobre los BRICS, los economistas de la banca remarcaban la necesidad de que estos países mantuvieran una política de apertura de los flujos comerciales y financieros, acompañados de un contexto macroeconómico estable con baja inflación, como condición de posibilidad para lograr un desarrollo exitoso. La apertura al comercio y la inversión extranjera directa, sostenía la banca, proporcionaría accesos a los insumos importados, nuevas tecnologías y mayores mercados; y, posteriormente, realizaban una advertencia, afirmando que

cada uno de los BRICs se enfrenta a retos muy importantes para el mantenimiento de su desarrollo. Esto significa que existe una posibilidad importante de que nuestras proyecciones no sean cumplidas, ya sea por una mala política interna o por mala suerte (Wilson y Purushothaman, 2003, p. 16).

Como señala Yory (2014, p. 30):

(...) el origen de los llamados grupos territoriales emergentes es necesario buscarlo en las motivaciones de los Bancos y de las entidades financieras que, en el contexto de la crisis económica actual, derivada de la propia crisis del modelo económico capitalista neo liberal, no sólo los inventan, tratando de reciclar y salvar el modelo, sino que los promueven -a través de novedosas estrategias de marketing- asignándoles el ostentoso título de " economías del futuro ”.

Esto marca una de las aristas fundamentales del concepto de emergente. Si hoy se puede hablar de China, Brasil, India, Rusia y (en menor medida) Sudáfrica como nuevos polos de poder global, que emergen en el Siglo XXI intentando poner fin a la unipolaridad norteamericana, esto se debe en gran medida al crecimiento notable de su economía, producto del redireccionamiento de las inversiones de los grandes capitales transnacionales hacia esos países. Este cambio en los destinos de la inversión global no fue casual, sino que fue alentado y promovido por una de las bancas globales de inversión más importantes a nivel mundial. 
Pero, por otro lado, este proceso de emergencia de nuevos actores en el escenario global fue acompañado por una política de activa participación estatal en la economía, muy diferente a otros procesos de crecimiento que tuvieron en los años 90 países como Chile, México, Taiwán y Hong Kong, entre otros. Estos últimos tuvieron tasas sostenidas de crecimiento de su PBI mediante su adhesión a las políticas neoliberales de apertura al libre comercio internacional, mientras que al interior de los BRICS se llevó a cabo una lucha por reemplazar este paradigma de crecimiento por uno centrado en una economía de mercado, pero con fuerte presencia estatal en el diseño, planificación y ejecución de la política económica y social.

Son importantes aquí los aportes de Pelfini y Fulquet (2015, p. 112), quienes afirman que:

(...) el primer criterio para definir tal emergencia (...) , es registrar y comparar tasas de crecimiento. Sin embargo, este indicador se relaciona más bien con el concepto de mercado emergente. El término potencia o poder emergente, por su parte, agrega a esto la fortaleza política, medible, en primer lugar, en términos de supremacía militar, pero también de solidez institucional y como capacidad de liderazgo a nivel regional (dimensiones que permiten hablar más propiamente de poder emergente y menos de potencia, que tiene demasiada carga geopolítica y de seguridad).

Paralelamente, los BRICS también aumentaron sus índices de inversión hacia otros mercados,al pasar de 7.000 millones de dólares en 2000, lo que representaba un 1\% del total mundial, a u\$s 126.000 millones en 2012, un $9 \%$ a nivel mundial. Estas inversiones tuvieron como destinatarios, principalmente, a otros mercados emergentes, con un $42 \%$ del total.

Retomando a Gobbi y Grande (2013, p. 9), se observa que:

(...)este perfil de origen de la IED distingue a China respecto de otros países en desarrollo, por la poca representación que tienen en el stock de inversiones los países del G-7, sólo el 20\% del total. El vertiginoso cambio en la pauta exportadora de China señala también que no es sólo una plataforma de ensamblaje, unida a las cadenas de valor global que son controladas por las grandes empresas multinacionales, sino que experimenta un proceso de profundización de su estructura industrial de la cual participan activamente las empresas de capital local.

Los BRICS-emergentes son generadoras del $45 \%$ de las exportaciones mundiales, poseen el $75 \%$ de las reservas de los bancos centrales, consumen más de la mitad de la energía mundial y han sido responsables del $80 \%$ del incremento de la demanda de petróleo durante los últimos años (Steinberg, 2008). Para comienzos del siglo XXI, los países desarrollados representaban un 63\% de los bienes y servicios producidos a nivel mundial, mientras que las economías emergentes explicaban solo un 20\%. Si se les suma el conjunto de los países en desarrollo, los países emergentes, para los años 80, explicaban el 36\% de la riqueza global generada, mientras que las economías avanzadas representaban el 64\% restante. Para 2014, mientras que las economías centrales explicaron el 43\%, las economías emergentes lo hicieron en un 57\% del PBI mundial. Fue en 2007 cuando las economías emergentes y las economías avanzadas se repartieron en partes iguales la creación mundial del PBI.

Es importante señalar que el crecimiento económico de los países emergentes, aunque con matices, produjo en ciertos sectores de su estructura económica una suerte de desarrollo industrial ligado al complejo estatalestratégico, a la vez que la incorporación de grandes masas de población, hasta entonces en situaciones de pobreza, a la cadena productiva a partir de la recuperación del trabajo, permitió una expansión del consumo interno, que contribuyó a generar un aumento considerable en el valor de las materias primas y commodities a nivel mundial. Como señalan Gobbi y Grande (2013), los precios de las materias primas se mantuvieron relativamente estables desde 1988 hasta 2002, cuando inician un ciclo de valorización en el marco de la crisis que las llevaron a cuadruplicar su nivel. Esta tendencia alcista sufre una breve interrupción a fines de 2008, y vuelve a retomar el crecimiento, principalmente en productos como el petróleo, el cobre, el níquel, la soja y el arroz. Como señalan los autores

entre los factores a considerar se encuentra, en primer lugar, el aumento del ingreso mundial, impulsado por la transformación estructural de países como China e India, que se plasma en un cambio en el patrón de consumo de su población, que presenta alta elasticidad en el ingreso de la demanda de bienes primarios (Gobbi y Grande, 2013, p. 10). 
A su vez, puede observarse el sostenido aumento de la demanda de minerales, metales y otros productos que sirven de insumos para la nueva estructura industrial de las economías emergentes. "Este escenario de ampliación de la demanda agregada de 'commodities' tuvo un evidente efecto favorable en el aumento de las exportaciones primarias de los países en desarrollo, emergentes y desarrollados ricos en recursos naturales" (Gobbi y Grande, 2013, p. 11). El redireccionamiento de las inversiones de los grupos transnacionales, de este modo, estuvo dado también por el aumento significativo de la demanda interna, producto del crecimiento de la capacidad de consumo de los sectores populares y de la clase trabajadora en los bloques emergentes.

En este proceso de disputa por la resultante del proceso de emergencia de estos nuevos actores (emergencia subordinada a los capitales transnacionales o emergencia comandada por la planificación estatal), la crisis financiera global resultó un punto de bifurcación en tanto posibilitó la coordinación conjunta de este grupo de países reclamando la necesidad de una transformación del orden mundial e impulsando el desarrollo económico, político y social mutuo por fuera de los designios de las instituciones políticas y económicas tradicionales.

Juncal (2014) afirma que los BRICS fueron posicionándose de tres maneras diferentes frente a la coyuntura mundial actual: por un lado, reclamando a los "países desarrollados" una mayor incidencia en los organismos de crédito internacional (FMI y Banco Mundial), así como también una democratización de las instituciones políticas globales (Consejo de Seguridad de la ONU); en segundo lugar, generando iniciativas conjuntas (no exclusivamente en materia económica, sino también política y social) para poder consolidar la articulación entre los socios o comenzar a suplir la falta de respuesta de los países desarrollados a los reclamos; y, en tercer lugar, pretendiendo establecer un liderazgo de los países emergentes a nivel mundial a partir de la creación de instituciones propias.

\section{La construcción de una Nueva Arquitectura Financiera Internacional MultipolaR}

Una de las reivindicaciones que aparece decisivamente en los discursos de los BRICS es la necesidad de dar paso a un nuevo mundo multipolar. Zambrano Jáuregui (2012) entiende al multipolarismo como una forma de toma de decisiones en donde el consenso y la negociación con varias partes son esenciales. El propósito del multipolarismo sería, para el autor, "establecer reglas de comportamiento internacional de mutua satisfacción para los países, así como el desarrollo de instituciones que favorezcan la cooperación internacional" (p. 48). Este nuevo orden mundial implicaría la coexistencia de polos de poder que no sólo se limitarían al plano de lo económico-financiero, sino polos-regiones de poder político, cultural y social.

El planteo de la necesidad de una Nueva Arquitectura Financiera Internacional, en tanto complejo jurídico, institucional y normativo que rige las relaciones financieras y monetarias públicas y privadas a escala internacional, será uno de los ejes articuladores del BRICS para la construcción de un mundo multipolar. La Vieja Arquitectura Financiera Internacional (Páez Pérez, 2011) hace referencia al ordenamiento económicofinanciero creado por las potencias vencedoras de la Segunda Guerra Mundial en Bretton Woods, y comprendía la complementariedad entre tres instituciones principales: el FMI, el Banco Mundial y la OMC. La liberalización de los tipos de cambio que se produjo luego de la inconvertibilidad del dólar en oro anunciado por Nixon en 1971, significó la profundización del rol del FMI como organismo de seguimiento de la deuda contraída con bancos privados y la administración de programas de "salvataje" y ajustes.

Entre las diferentes Cumbres anuales del BRICS, los miembros del bloque hicieron un particular hincapié en la necesidad de "democratizar" los instrumentos económico-financieros heredados de los acuerdos de Bretton Woods. Esto significaba, en lo concreto, modificar la correlación de fuerzas entre los distintos proyectos a nivel global, al reclamar un protagonismo cada vez más fuerte en los espacios de decisión global. 
Con este propósito, los BRICS-emergentes crearon una serie de instrumentos financieros para ampliar sus grados de soberanía, entre los que se encuentran el Banco Asiático de Inversiones en Infraestructura (BAII), el Nuevo Banco de Desarrollo del BRICS y el Fondo de Reservas de Contingencia. Tanto el BAII como el NBD-BRICS constituyen herramientas clave para apuntalar los déficits de infraestructura que tienen los países emergentes, mientras que el FRC permite reducir sustantivamente la volatilidad cambiaria sobre los flujos de comercio e inversión entre los miembros del bloque, y permite proteger sus economías, incluido sus monedas, ante los posibles ataques especulativos de las grandes potencias globales y grupos económicos financieros transnacionales.

La creación del Fondo de Reservas de Continencia y del Nuevo Banco de Desarrollo puso de manifiesto una aceleración de este proceso de disputa por una nueva arquitectura financiera, frente al escaso avance en la reforma de la arquitectura financiera global-unipolar, luego de la crisis de 2008. En esta línea, Noyola Rodríguez (2014) afirma que estos dos instrumentos constituyen las semillas de una nueva arquitectura financiera global, que sienta las bases para un nuevo orden mundial con la mira puesta, por un lado, en ampliar la representación de nuevos actores emergentes en la toma de decisiones globales y, por otro, en contrarrestar la unipolaridad norteamericana y europea en los mercados financieros internacionales.

Otro de los ejes centrales que sobrevoló los distintos debates en torno a la necesidad de una nueva arquitectura financiera internacional es la decisión de abandonar el dólar y el euro como monedas de intercambio en el comercio internacional y de reserva. En distintos momentos, los presidentes del BRICS hicieron hincapié en la importancia de impulsar las transacciones en las divisas de las naciones integrantes del bloque, con una infraestructura financiera propia que permita eliminar el uso de monedas externas a las de sus países. Esto constituye no solo un intento de excluir al dólar estadounidense y al euro para reducir costos financieros y cambiarios, sino como una estrategia para logar mayores grados de soberanía.

Disputarle al dólar su condición de moneda de referencia global implica dar un golpe en uno de los puntos nodales de la vieja arquitectura financiera. El 30 de noviembre de 2015, el FMI aceptó finalmente a la moneda china (el yuan) para ser parte de la cesta de monedas DEG y, el 18 de diciembre, el Congreso de los Estados Unidos dio el visto bueno para acelerar el proceso de reforma del sistema de votación del FMI, otorgándole más peso a las nuevas potencias emergentes. La nueva composición de los Derechos Especiales de Giro, asignó al Yuan el 10,92\% de su proporción, ubicándolo en tercer lugar, por debajo del dólar y el euro.

\section{LA CONSTRUCCIÓN DE UNA NUEVA ARQUITECTURA PRODUCTIVA}

Este proceso de lucha por llevar adelante una estrategia de desarrollo soberano tiene como una de sus principales políticas la construcción de la Nueva Ruta de la Seda, como modelo de cooperación económica, política, social y cultural entre Asia, frica y Europa.

El Cinturón Geoestratégico de la Ruta de la Seda fue anunciado en 2013 por el presidente chino Xi Jinping, afirmando que el mismo contemplaría proyectos de infraestructura para conectar por tierra y mar el sudeste asiático con Asia Central, Europa y reforzar la cooperación con África. El proyecto se compone de dos rutas, conocidas como "Un Cinturón, Un Camino". La ruta terrestre es llamada "Cinturón Económico Ruta de la Seda", y une el centro de Asia con Rusia y Europa. La ruta marítima, a su vez, es denominada "Ruta Marítima de la Seda del Siglo XXI” y pasa por el Pacífico occidental y el Océano Índico (Fotografía N. ${ }^{\circ} 1$ ). 


\section{FOTOGRAFÍA N.ำ.}

La Nueva Ruta de la Seda. Por tierra, las alternativas del Cinturón Económico de la Ruta de la Seda. Por agua, la Ruta Marítima de la Seda del Siglo XXI. Los trayectos son estimados, ya que la propuesta del OBOR es de alcance mundial.

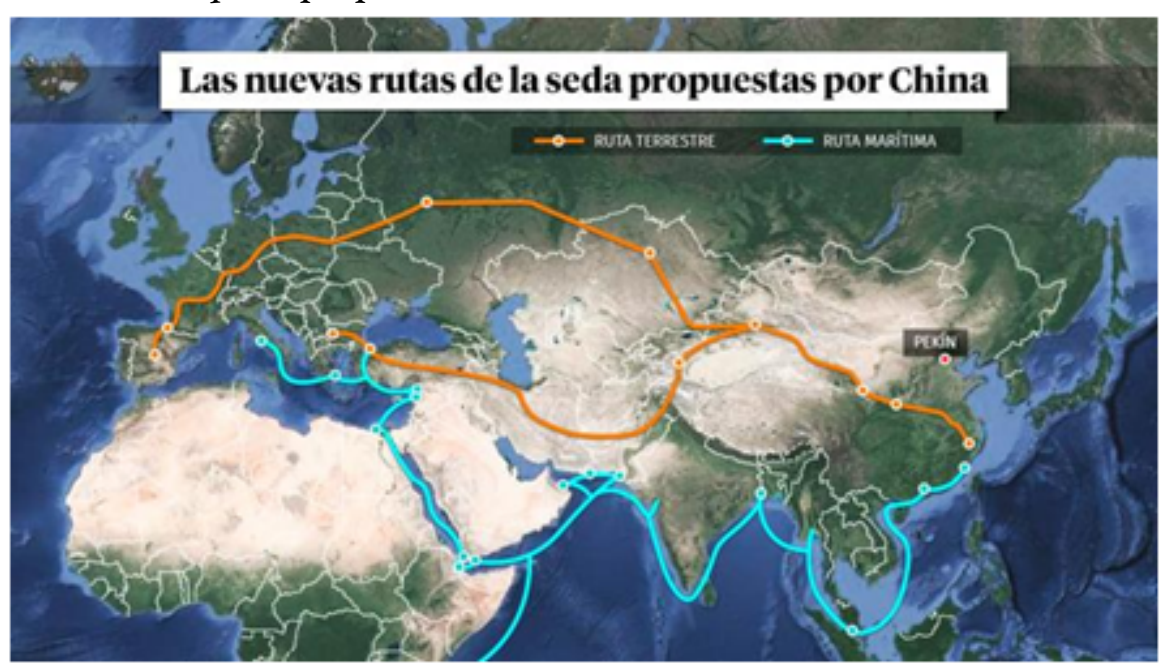

Fuente: Diario La Vanguardia. http://www.lavanguardia.com/economia/201 70619/423355535498/ruta-de-la-seda-china-imperio-del-centro-global.html.

La Ruta de la Seda es esencialmente un proyecto productivo-industrial, ya que implica la construcción de vías ferroviarias, carreteras terrestres, rutas aéreas y marítimas, proyectos energéticos, parques industriales y puertos para el desarrollo soberano de los países involucrados. La propuesta de la nueva Ruta de la Seda viene a confirmar la consolidación de un proyecto basado en el trabajo y en la economía real, motorizado por la principal potencia económica en ascenso, la República Popular China, y sostenido por y articulando un conjunto de bloques regionales emergentes que propician un nuevo orden mundial multipolar. De esta manera, se profundiza el hecho de que el centro de gravedad unipolar con asiento en el complejo financiero, industrial y militar angloamericano, dominante hasta 2001 y luego en declive, comienza a desplazarse hacia nuevos polos de poder asentados en las zonas emergentes. En este sentido, Giaccaglia (2016,p. 8) afirma que "Asia se ha convertido en el nuevo centro de gravedad mundial (...) un despertar con el advenimiento de nuevas ideas y, por ende, de una nueva forma de pensar el mundo”.

A grandes rasgos, los proyectos de infraestructura más importantes de la Ruta de la Seda son: ${ }^{5}$

1. el Corredor Económico China-Mongolia-Rusia;

2. el Corredor China-Asia Central-Asia Occidental, un conjunto de vías marítimas y terrestres que conectan Kazajstán, Azerbaiyán, Armenia y Turquía;

3. el Corredor península Indochina-China, compuesto por tres rutas: la primera, desde Kunming hasta Laos y Tailandia; la segunda, hacia Vietnam y Camboya; y, finalmente, la ruta hacia Myanmar;

4. el Corredor China-Pakistán;

5. El ferrocarril hacia Irán, puerta de entrada a Medio Oriente;

6. La vía férrea que une la ciudad china de Yiwu con Madrid y Londres, de 12 mil kilómetros, la vértebra comercial que conecta Asia y Europa;

7. La Ruta Marítima del Norte/Ruta de la Seda Polar, una ruta que recorre el mar del Norte a través de 7.300 millas náuticas uniendo los puertos del extremo este de Rusia, pasando por el estrecho de Bering, hasta el puerto de Bilbao en España. 


\section{LOS PLANTEOS ESCÉPTICOS SOBRE EL BRICS.}

Desde distintos ensayos de opinión y artículos científicos, en los últimos años, se intentó instalar el clima de que los BRICS se habían rápidamente agotado, ya sea por la baja en el ritmo de crecimiento de sus economías, por las diferencias internas entre los países miembro, o por la falta de nuevas iniciativas concretas que tradujeran en política práctica lo que proclamaban en el discurso.

La reducción de la tasa de crecimiento y de las exportaciones de China y de la India que comenzaron a manifestarse desde 2012, con efectos negativos en las balanzas comerciales de países como Brasil, Argentina, Chile, Colombia o Perú, sirvieron de base para sustentar la idea de que nos encontramos en la etapa final de un ciclo de expansión iniciado en 2000, que se manifestaría en la vuelta de los "stop-and-go" que caracterizaron durante décadas a los países subdesarrollados.

A partir de esto, en 2008 la propia Goldman Sachs comenzó a hablar de nuevos países que superarían a los BRICS como polos de inversión global, especialmente los llamados “MIST": México, Indonesia, Corea del Sur y Turquía. Para la banca, los MIST eran economías que duplicaron su tamaño en los últimos diez años y que, a diferencia de los BRICS, no han sido afectadas por las crisis económicas, tanto de Estados Unidos como la crisis europea. Esta política se vio reflejada en las inversiones que realizó el grupo: mientras que el fondo de inversión MIST tuvo una rentabilidad del 12\%, el fondo de inversión destinado a los BRICS se mantuvo en el 1,5\% (BRIC vs. MIST, 2012). Sin embargo, el propio presidente de Gestión de Activos de la GS, y creador del término BRIC, Jim O’Neill, continuó sosteniendo la vigencia del concepto, al afirmar que

me hace gracia cuando los medios de comunicación dicen que la historia de crecimiento de los BRIC ha llegado a su fin. A mí me resulta muy divertido leer esta clase de historias. Aparte de su aversión al término BRIC, no tengo claro en qué fundamentan sus opiniones (Jim O’Neill se retira, 2013).

El periódico británico The Independent incluso fue más duro con los BRICS; en palabras de su editorialista Chris Blackhurst, afirmó que

el viento fue quitado de sus velas y la idea de que puedan reemplazar a los Estados Unidos parece más lejos que nunca. Su incapacidad de gobernarse a sí mismos, de producir sanas y seguras políticas económicas, de poner sus negocios en un mundo golpeado ha demostrado su fracaso (Blackhurst, 2015).

La cadena CNN también se manifestó en el mismo sentido: "Los mercados emergentes, largamente vistos como los niños mimados de la economía global, se han desplomado" (El brillo de los países BRICS se apaga, 2013). Ruchir Sharma, jefe de mercados emergentes de la banca Morgan Stanley, afirmó que los BRICS ya no eran destinos interesantes para la inversión y que, en la actualidad, "mis mejores apuestas son países donde están ocurriendo reformas económicas y donde exista un nuevo liderazgo político. Eso está sucediendo en Filipinas, México y Pakistán” (El brillo de los países BRICS se apaga, 2013). En otra entrevista, el ejecutivo de la Morgan Stanley afirmó que "los BRIC nunca fueron y nunca serán un bloque económico o político (...) Fueron estrellas inesperadas” (Rodrigues, 2013).

Un dato relevante en este sentido es que, en octubre de 2014, la Reserva Federal norteamericana (FED), comandada por Janet Yellen, comenzó a implementar el fin del programa de estímulos monetarios (hecho que ya había sido anunciado por el expresidente de la FED, Ben Bernanke en octubre de 2013), una política que consistía en la compra compulsiva de bonos gubernamentales "basura" y de obligaciones hipotecarias que no estaban pudiendo ser pagadas por los deudores, y de préstamos a bajas tasas de interés (que iban del 0 al $0,25 \%)$, que fueron a parar principalmente a los mercados emergentes. Esta medida, que había sido puesta en marcha luego de la crisis financiera de 2008 con la excusa de reactivar la economía de los Estados Unidos, significó la inyección de casi 3,7 billones de dólares a los países emergentes. Como señala la Banca de Inversión Standard Ch.(Lopes, 2014): 
(...) la política de la Reserva Federal ha incentivado que llegase una fuerte oleada de dinero a los países emergentes. Imagínese que pide prestado dinero por nada. Sí, por nada, porque las tasas de interés en Estados Unidos están al 0-0,25 por ciento. Y quiere invertirlo en algo que le dé mucha rentabilidad. La renta variable emergente y la renta fija están a niveles históricamente atractivos (...) Como quiere sacar la mayor rentabilidad posible elige como destino de su inversión los emergentes, como muchos otros inversores.

Como señalan Gobbi y Grande (2013), esta política de “expansión cuantitativa” tuvo tres consecuencias centrales para los mercados emergentes: en primer lugar, significó la entrada de capitales motivados por la búsqueda de rentabilidad asociada al diferencial de tasa de interés; en segundo lugar, produjo la apreciación relativa de sus monedas, debido al efecto devaluatorio sobre el dólar estadounidense; y, en tercer lugar, mejoró los términos de intercambio, al aumentar el precio de las commodities a nivel mundial.

Si bien estos factores monetario-financieros impulsaron el crecimiento del PBI de las economías emergentes en términos nominales, las colocaron a su vez en una situación de vulnerabilidad a shocks externos (...) En efecto, la fragilidad de esta coyuntura se verificó ante el anuncio del Presidente de la Reserva Federal de Estados Unidos, de que la política de expansión cuantitativa sería moderada (p. 13).

Por otro lado, una de las críticas más importantes al BRICS fue la decisión de los países miembro, en 2011, de incluir a Sudáfrica como miembro pleno; en este sentido, el creador del acrónimo, Jim O’Neill, afirmó que "su economía es tan pequeña, que no se justifica su consideración en la misma esfera económica (...) nisiquiera es la mayor economía de África subsahariana, que es Nigeria” (O’Neill, 2014)

\section{REFlexiones FinALES}

La aparición de los BRICS en la escena internacional sin dudas marcó una reconfiguración de las relaciones de fuerzas globales, y no solo puso en cuestión y en crisis el ordenamiento global que había sido hegemónico luego de la Segunda Guerra Mundial, sino que empezó a proyectar para el fututo la posibilidad de un nuevo mundo: un mundo multipolar.

Aunque el bloque de países emergentes ya es un jugador de peso en el tablero global, aún no se puede saber con certeza cuál es en concreto el proyecto de nuevo mundo planteado por los BRICS. Esto puede deberse a tres cuestiones: en primer lugar, los BRICS, si bien aparecieron como acrónimo en 2001, entraron en la escena global recién en 2009 con su primera Cumbre de mandatarios; es decir, con solo nueve años de vida, resulta difícil todavía visualizar en profundidad su proyecto. Pero, en segundo lugar (y quizás más importante aún), el BRICS no es un bloque homogéneo y monolítico, sino que existen distintos proyectos estratégicos que disputan en su interior por encabezar la cruzada por la conformación de un nuevo mundo. Finalmente, en tercer lugar, la construcción de un nuevo escenario global va a depender de cómo se atraviesen estos momentos de crisis y de cómo se subordinen o no los actores que hoy son dominantes.

Sin embargo, más allá de lo expuesto más arriba, se puede afirmar que hoy dominantemente en el mundo han ganado terreno aquellos actores que plantean la necesidad de superar la vieja hegemonía trilateral Estados Unidos-Unión Europea-Japón, donde estos tres actores se repartían el mundo y actuaban unilateralmente atribuyéndose el rol de potencias centrales.

Este proceso de "desoccidentalización” (Mignolo, 2011) nos abre, a la otrora países subdesarrollados, hoy emergentes, nuevos e importantes desafíos. Porque, como se expuso en el trabajo, la conformación de los bloques multipolares soberanos resulta estratégica para concretar el sueño de la Patria Grande Latinoamericana, como una más dentro de muchas Patrias Grandes soberanas a nivel global. Las preguntas de Bruckmann (Callisaya, 2018) son sumamente pertinentes, entonces, para pensar los desafíos de nuestra región:

¿Qué cosa va a hacer América Latina? ¿Va a continuar reproduciendo su inserción dependiente y subordinada en el sistema mundial como exportadora de materia prima sin valor agregado? O por el contrario, va a aprovechar esta oportunidad 
histórica para agregar valor agregado a la exportación de estos recursos naturales a hacer cadenas de valor regionales, a industrializar, a supeditar esta comercialización de recursos naturales a transferencia tecnológica, a colaboración científica, industrial. Este es el desafío.

\section{REFERENCIAS BIBLIOGRÁFICAS}

Blackhurst, C. (19 de agosto de 2015) It's all over for the Brics countries now - upstarts are taking the initiative. The Independent. Recuperado de: https://www.independent.co.uk/voices/its-all-over-for-the-brics-countries-nowupstarts-are-taking-the-initiative-10462624.html.

"BRIC vs. MIST" (1 de diciembre de 2012). Diario La Razón de Mexico. Recuperado de: https://www.razon.com.m $\mathrm{x} /$ negocios/bric-vs-mist/.

Callisaya, S. (2018). ¿Cuál es la estrategia en América Latina? Asia en la economía mundial [entrevista a Mónica Bruckmann]. Portal ALAInet. Disponible en https://www.alainet.org/es/articulo/193106. [Consultado en línea el 13/06/2018].

"El brillo de los países BRICS se apaga" (23 de agosto de 2013). Diario CNN Expansión. Recuperado de: https://exp ansion.mx/economia/2013/08/22/mas-problemas-en-los-mercados-emergentes.

Formento, W. y Merino G. (2011). Crisis financiera global. La lucha por la configuración del orden mundial. Buenos Aires: Ediciones Continente .

Formento, W. (2015). Crisis financiera global y oportunidad histórica para los pueblos del mundo. En Crisisfinanciera global, su desarrollo e impacto social (pp. 3-11). Buenos Aires, Centro de Investigaciones en Política y Economía .

Giaccaglia, C. (2016). Asia: turbulencia. Revista Voces en el Fenix, 7(56).

Gobbi, H. y Grande, J. (2013). La nueva gobernanza económica internacional. Revista Relaciones Internacionales, 45.

"Ins and Outs. Acronyms BRIC out all over" (18 de septiembre de 2008). The Economist. Recuperado de: https://w ww.economist.com/special-report/2008/09/18/ins-and-outs.

“Jim O’Neill se retira” (6 de febrero de 2013). Diario Founds People. Recuperado de: https://es.fundspeople.com/ne ws/jim-o-neill-se-retira.

Juncal, S. (2014) El surgimiento de los BRICS y sus implicancias para el orden global. FLACSO-ISA Joint International Conference, Buenos Aires. Disponible en: http://web.isanet.org/Web/Conferences/FLACSO-ISA\%20Buenos Aires\%202014/Archive/1e84e2ef-a5b8-46a0-a173-94aa6a09abba.pdf. [Consultado en línea el 10/06/2018].

Katz, C. (2011). El ajedrez global de la crisis. Crítica e Sociedade: revista de cultura política. 1(3).

López, M. (1 de febrero de 2014) La crisis de los países emergentes y el 'cuento de los tres cerditos'. Diario El Economista de América. Recuperado de: https://www.eleconomistaamerica.com.ar/mercados-cotizaciones/noticias/55068 57/02/14/La-crisis-de-los-paises-emergentes-y-el-cuento-de-los-tres-cerditos.html.

Martins, C. E. (2014). El sistema-mundo capitalista y los nuevos alineamientos geopolíticos en el siglo XXI: una visión prospectiva. En M. Gandásegui, C. Martins y P. Vommaro (coord.),Soberania, hegemonía e integración de las democracias en revolución en América Latina. Quito: Editorial IAEN.

Mignolo, W. (2011).Geopolitica de la sensibilidad y del conocimiento. Sobre (de)colonialidad, pensamiento fronterizo y desobediencia epistémica. Disponible en http://marceloexposito.net/pdf/trad_mignolo_geopolitica. pdf. [Consultado en línea el 10/06/2018].

Noyola Rodríguez, A. (2014). Geopolítica del Banco BRICS . Publicado en línea en Red Voltaire. Disponible en: htt p://www.voltairenet.org/article184789.html [Consultado en línea el 10/06/2018].

O’Neill, J. (26 de julio de 2014) Is the BRICS rise over?.Bruegel. Recuperado de: http://bruegel.org/2014/07/is-th e-brics-rise-over/.

Páez Pérez, P (2011). Otro modelo financiero ya está en marcha en América Latina . Transnational Institute (TNI).Disponible en https://www.tni.org. [Consultado en línea el 12/06/2018]. (Error 12: El enlace externo www.tni.org debe ser una url) (Error 13: La url www.tni.org no esta bien escrita) 
Pelfini, A. y Fulquet, G. (comps.).(2015). Los BRICS en la construcción de la multipolaridad ¿Reforma o adaptación?. Ciudad Autónoma de Buenos Aires: CLACSO.

Rodrigues, J. (2013). The BRICs never were and never will be an economic or political bloc. A conversation with Ruchir Sharma . Disponible en: http://janelanaweb.com/novidades/\%C2\%ABthe-brics-never-were-and-never -will-be-an-economic-or-political-bloc\%C2\%BB-ruchir-sharma/ [Consultado en línea el 12/06/2018].

Sader, E. (2014a). Un mundo multipolar . Publicado en: https://www.cartamaior.com.br/?/Blog/Blog-do-Emir/Um -mundo-multipolar/2/30559. [Consultado en línea el 09/06/2018].

Sader, E. ( 19 de julio de 2014). Brics: la hegemonía alternativa .Página/12 .

Steinberg, F. (2008). El impacto de las potencias emergentes en la economía mundial. Madrid: Real Instituto Elcano .

Turzi, M. (2011). Mundo BRICS: las potencias emergentes. Buenos Aires: Capital Intelectual .

Wilson, D. y Purushothaman, R. (2003). Dreaming with BRIC s: The Path to 2050. Global Economics Paper, 99, Goldman Sachs. Disponible en [Consultado el 12 de junio de 2018].

Yory, C. (2014). El grupo BRIC y el 'efecto roquefort': una aproximación a la noción de región-emergente en el contexto de la globalización. GEOgraphia, 14(27), 21-45.

Zambrano Jáuregui, C. (2012). El multilateralismo actual: crisis y desafíos. Revista de Relaciones Internacionales, Estrategia y Seguridad, 8(1).

\section{Notas}

1 En el G7 comenzó a funcionar en 1973, en principio como una reunión de Ministros de Economía y Finanzas de las grandes potencias centrales, y luego como una Cumbre anual de presidentes de las mismas (Alemania, Canadá, Estados Unidos, Francia, Italia, Japón y Reino Unido).

2 Entre 2001 y 2009 se utiliza el término BRIC, acrónimo que comprendía a Brasil, Rusia, India y China. En 2011, los miembros del grupo aprobaron la incorporación de Sudáfrica, por lo que adoptó su nombre definitivo de "BRICS".

3 Retomamos aquí la definición de Yory (2014) quien define al Estado en tanto construcción social procesual autoorganizada, es decir, como una construcción territorial definida no solamente a partir de una forma de ocupación espacial, sino una construcción (lucha) hecha por actores concretos (proyectos estratégicos en pugna), que en su lucha van definiendo procesualmente la forma que toma el Estado-Nación.

4 El término Next-11 fue creado por la Goldman Sachs en 2005 para referirse a nuevas economías del futuro que vendrían un escalón por debajo del BRICS: Bangladés, Corea del Sur, Egipto, Filipinas, Indonesia, Irán, México, Nigeria, Pakistán, Turquía y Vietnam. Los criterios establecidos por la banca para agrupar estos países fueron: estabilidad política, apertura comercial, sus índices de Inversión Extranjera Directa, su calidad educativa y su estabilidad macroeconómica.

5 El 14 y 15 de mayo de 2017, Xi Jinping organizo el Primer Foro "Una Franja Una Ruta para la cooperación internacional”, donde se convocaba a distintos mandatarios a ser parte de la iniciativa de la Ruta de la Seda. A la misma asistieron 28 presidentes y primeros ministros, cien representantes ministeriales y unos 1.200 delegados de más de 130 países de todo el mundo, además de la directora gerente del FMI, Christine Lagarde, el secretario general de las Naciones Unidas, Antonio Guterres y el presidente del Banco Mundial, Jim Yong-Kim. 